Brit. J. vener. Dis. (1956), 32, 140.

\title{
THE CHALLENGE OF SYPHILIS TO SCIENCE*
}

\author{
BY \\ EVAN W. THOMAS $\dagger$ \\ Venereal Disease Consultant, New York State Department of Health, Albany, N.Y., U.S.A.
}

Thanks to the antibiotics, the treatment of syphilis, with possible rare exceptions, is now very simple. Hence, the general practitioner may feel justified in relegating it among the minor problems of his profession. Yet, neither the practising physician nor the scientific investigator has as yet fathomed the secrets of the $T$. pallidum or of the varied and complex responses of the human host. This fact is of more than academic interest. The lag between our understanding of syphilis and its treatment suggests that complete control of the infection cannot be anticipated without further knowledge of the treponeme and of the immune reactions in man.

No other common infectious disease presents the pathologist with so many complex, controversial, and unsolved problems as syphilis. The variety and uniqueness of some of the phenomena defy explanation by the general principles and theories that have proved useful in understanding most other infections. This statement, if true, as I believe it is, presents a challenge to the scientist concerned with the understanding and integration of immunological mechanisms such as can be provided by no other single infectious disease.

Much has been accomplished in the past century in recognizing and classifying the protean manifestations of syphilis. In reading the literature, we are impressed by the diagnostic acumen of such men as Ricord, Fournier, Neisser, Hutchinson, and others. We are indebted to these earlier physicians for much of our present knowledge of the disease. Yet this should not blind us to the fact that the literature on syphilis is replete with discrepancies, controversies, and even contradictions. Careful analysis of cases presented in the voluminous literature suggests that sometimes diagnoses were influenced by a particular belief or theory rather than by ruling out other possibilities. This is understandable because, paradoxically, the less we know about the mechanisms underlying phenomena, the more dogmatic and influential accepted beliefs tend to be.

\footnotetext{
* Received for publication July 5, 1956.

† Formerly Director, Syphilis Service, Bellevue Hospital, and Professor of Clinical Medicine (Syphilis Section), New York University College of Medicine, New York City.
}

In science it is axiomatic that beliefs must be supported by good evidence. Unfortunately, in syphilis, good evidence for the solution of numerous problems has been difficult, if not impossible, to obtain. In medicine, as in science, good evidence consists of the most logical probabilities. The less exact the science, the less exact the probabilities. Since many of the phenomena of syphilis have fallen far short of explanation by the more exact sciences, we have had to make the most of such evidence as has been available. This necessity has at times led us astray and, once beliefs become established by years of repetition, we tend to accept them and cease to look for other possibilities until. forced to do so by overwhelming new evidence.

Thus, not much more than a decade ago, reinfection after treatment of early syphilis was regarded by most authorities as unlikely and even impossible. Only the advent of rapid treatment and its careful evaluation brought conviction not only that re-infection was possible after treatment of early syphilis, but also that it frequently occurred.

A similar example may be found in the exaggerated numbers of so-called Jarisch-Herxheimer reactions which were reported after the treatment of cardiovascular syphilis. With the exception of general paresis, where treponemes may be found in great abundance in the cerebral cortex, Herxheimer reactions are uncommon in late syphilis. However, the possibility, if not the probability, of this reaction afforded a handy peg on which to hang otherwise unexplained accidents after the treatment of cardiovascular syphilis. Experience with penicillin now indicates that many of the accidents formerly reported as Herxheimer reactions were caused by something else. Presumably the Herxheimer reaction is due to the rapid killing of many treponemes, but this does not explain the mechanism of the reaction, which is one of the numerous relatively unique, unexplained phenomena of syphilis.

Different but still pertinent examples of the difficulties encountered in syphilis are the amazing discrepancies found in the literature on the frequencies of late lesions. An entire paper could be devoted to this subject alone. Here I merely wish 
to mention that, from the Gjestland report of untreated syphilis in Oslo (Gjestland, 1955) and from Rosahn's review of autopsies in New Haven (Rosahn, 1947) demonstrable late lesions of syphilis have been observed in only some 30 to 40 per cent. of untreated cases. These findings are in agreement with impressions obtained from my own experience. But, when we turn to the literature for additional information, we find frequencies at great variance with each other as well as with the foregoing estimate.

Stokes reported late bone and joint lesions in 57 per cent. of males and 43 per cent. of females in a series of 239 late syphilitics in the Mayo Clinic (Stokes, Beerman, and Ingraham, 1944). He quoted Buchman and Lieberman as reporting similar lesions in 61 per cent. of a somewhat smaller series of male patients. Needless to say, if bone and joint lesions were that frequent in series supposedly representative of first-rate clinics in the 1920s and 1930s, the frequency of all late lesions must have been higher. Pathologists during the first 30 years of the 20th century have reported the frequency of cardiovascular syphilis at autopsy in a variety of series, most of which, it is true, are not claimed to be representative of syphilis in a general population. The reports that have come to my attention give frequencies varying from 19 per cent. or lower to 70 or 80 per cent. The latter percentage was reported by Langer (1926) from the massive material autopsied at no less a place than the Virchow Clinic in Berlin during the early years of this century. Even when due allowance is made for location, race, sex, age, treatment, and the selection of material, such discrepancies are disturbing. Since many of the individuals in the large Virchow Clinic series must have had some mercury treatment, if the data are correct and representative, one might easily suspect that mercury may have done more harm than good.

I mention these discrepancies in reported data, not because they now have practical significance, but because, when I began to study syphilis, they were one of the first warnings to take nothing for granted about this strange disease until it was supported by my own experience. Unfortunately, my experience has left me with many unanswered questions, and it has led to at least one mistaken conclusion regarding infectious relapse in untreated syphilitics. The latter, since it can be corrected, is less important than the former. However, my incorrect conclusion raises some important questions which I should like to discuss as illustrative of the problems that still confront us.

Syphilis comprises almost two distinct diseasesthe early and late stages. The immunologic differences between the two stages are profound. Not only do the body tissues react to the invading organisms differently during the two stages, but the $T$. pallidum seems to be profoundly affected by passage from the early to the late stage, as evidenced by the paucity of organisms found after the early stage has ended. Questions about the mechanisms underlying these changes remain unanswered. However, from clinical experience and animal experiments, we know that immunity in the sense of resistance to infection is developed to a much less extent during the early than the late stage and that humoral antibodies, such as the treponemal immobilizing antibody, do not seem to provide significant protection against infection after syphilis has been cured. Evidence for this statement can be found in the reports of animal experiments (Magnuson, Thompson, and McLeod, 1951) and in an experiment with human volunteers at Sing Sing Prison in New York State (Magnuson, Thomas, Olansky, Kaplan, De Mello, and Cutler, 1956). In the latter experiment, all of the eleven patients previously treated for early syphilis were re-infected by inoculation with virulent $T$. pallida of the Nicols strain, and under 50 per cent. of 33 patients previously treated for late syphilis showed any physical or serologic evidence of infection by the inoculation. All volunteers previously treated for late syphilis had positive TPI tests when inoculated. Although, in general, those with the highest STS and TPI titres showed no evidence of infection by the inoculation, one volunteer, presumably previously treated for late latent syphilis, developed a dark-field positive lesion at the site of inoculation and his TPI titre before inoculation was the highest found in any of the previously treated patients.

The fact that the early stage provides much less "immunity" to re-infection than the late stage makes it important to determine, if possible, the duration of the early stage. One of the means of arriving at some idea of the duration of this stage is to note the time during which infectious relapses occur.

In my experience, I have never obtained a history of relapsing secondary lesions from an untreated patient, and experience with relapses after treatment led me to believe that infectious relapse rarely occurs more than 2 years after the last treatment. Relying on this experience, I had the temerity to state (Thomas, 1949) that I did not believe that infectious relapse occurs in the absence of treatment and that the duration of untreated early syphilis was probably not more than 2 years. The folly of making general conclusions solely from one's own experience has been brought home to me by Gjestland's admirable and painstaking review of the Oslo series of patients 
diagnosed as having early syphilis in Boeck's clinic (Gjestland, 1955). Gjestland found that 25 per cent. of 1,035 patients relapsed without treatment. Among those who relapsed, 20 per cent. had multiple relapses, 70 per cent. relapsed within 2 years, and twelve instances of first relapse and fourteen of multiple relapses were observed between $2 \frac{1}{2}$ and 6 years after infection.

These figures surprise me. I accept them as evidence of relapse in untreated infections, but I wonder if the very late relapses would have been dark-field positive. I also wonder if Gjestland has proved his conclusion that "even inadequate treatment protects against relapse". Perhaps it depends on how inadequate the treatment is. In my experience, even very small amounts of penicillin given over 7 days resulted in fewer relapses than were noted after two injections of an arsenical drug when injections were given at weekly intervals. Moore's (1941) finding that 65 per cent. of patients, who receive only one to four injections of an arsenical drug, later develop relapses may be an exaggeration, but, if we cut the percentage in half, it is still higher than 25 and suggests a definite relationship between poor treatment and relapse. So far as I can determine, however, no data are sufficiently reliable to settle this question. My purpose is not to pursue a controversy over the frequency of relapse following poor treatment. Rather, I wish to discuss briefly why and how relapse occurs.

Chesney (1927) suggests that relapse is due to an increased resistance of the $T$. pallidum to immune factors in the host. In other words, healing of early lesions is caused by inhibition of the organisms which subsequently regain their ability to multiply freely. This may be due to a rhythmic change in the resistance of the $T$. pallidum or to an alteration in the inhibiting mechanisms of the host. In either case, poor treatment should assist in inhibiting the organisms temporarily, and it also might interfere with the development of immune factors in the host, thus favouring relapse. But we cannot stop with this possibility alone. We need to ask whether or not the skin and mucous membranes are reactive to the $T$. pallidum during the interval between the healing of lesions and relapse. If they are, we must assume that healing is associated with the death of organisms in the skin and mucous membranes and that relapse occurs when organisms again multiply freely and lodge within these tissues. If the skin and mucous membranes are not reactive in the latent period before relapse, we must assume that the healing of early lesions is associated with a lack or loss of sensitivity of the tissues and that the tissues become sensitized or resensitized when a relapse occurs. Such rhythmic alteration in tissue sensitivity could account for all relapses, regardless of treatment, but it would not explain why more relapses have been noted in association with very poor treatment than with less poor treatment (provided this difference is real and not based on mere impressions).

It is possible, as Gjestland suggests, that relapse in untreated individuals is caused by an immune mechanism different from the mechanism of relapse in treated patients. Possibly relapses in untreated patients are caused by an altered sensitivity of the tissues, while those associated with treatment are not. I wonder if patients capable of relapse after the healing of early lesions, especially after inadequate therapy, would not respond to virulent inoculation with a secondary papule.

The chief objection to this possibility is that, if the skin retains its sensitivity to the $T$. pallidum, response with a secondary lesion to inoculated $T$. pallida would be contrary to the rule of infection immunity; by which I mean that an untreated syphilitic, from the time of the original chancre onwards, will not react to inoculation with a chancre or presumably not with a dark-field positive lesion. It is conceivable, that this rule might not hold in all patients capable of relapse. Probably the skin of poorlytreated patients capable of relapse would be more likely to react to inoculated treponemes than would the skin of untreated cases, because non-curative treatment causes rapid healing of early lesions, and, in such cases, there may not be a correspondingly rapid alteration in the reactivity of the skin.

Such possibilities as this and the others I have mentioned are, of course, speculative. One means of investigating them could be to inoculate large numbers of individuals after the healing of early lesions in both poorly-treated and untreated cases. I doubt whether this was done satisfactorily in previous experiments which established the rule of infection immunity. It is even conceivable, I think, that some genital relapses following poor treatment might be due to superinfection. Whether or not this is possible, it would seem, theoretically at least, that very poor treatment might favour relapse. In cases with no relapse of early lesions, we cannot be sure how soon the immunological status of late syphilis is established. The early stage may end with the healing of lesions or some time later. Presumably, however, it occurs in most individuals within 2 years.

That immunity to infection is the rule in untreated latent syphilitics with no history of relapse is supported by the Sing Sing Prison experiment, where, five untreated volunteers with latent syphilis of unknown duration (probably more than 2 years) failed to respond to inoculation either by a lesion or by a change in the titres of their serological tests. 
This does not necessarily mean that the inoculated organisms were destroyed. Some of them may have survived and may have been treated by the body in the same way as those already present. I mention this possibility because I do not think we can determine the fate of heterologous treponemes in cases of so-called infection immunity until we know more about the $T$. pallidum and the mechanisms underlying the paucity of demonstrable organisms at the late stage.

In at least one type of late syphilis, immunity to superinfection is not the rule. Grin, from observations of endemic syphilis in Yugoslavia, concluded that many gummata were due to superinfection or re-infection, even though there was no history of previous gummata (Grin, 1953). In the Sing Sing Prison experiment, two previously well-treated volunteers with no history of gummata responded to inoculation with a gumma. Treatment of late syphilis had been initiated in both of these patients more than 10 years before inoculation, and neither had received treatment less than 2 years before inoculation, because by that time it was believed further treatment was no longer indicated. At what time during the course of their infection the particular type of skin sensitization, characteristic of gummatous reactions, had occurred is, of course, unknown. In view of their previous therapy, the gummata in the Sing Sing patients may have been due to re-infection rather than superinfection. In either case, the fact that inoculation resulted in a gumma indicates that this particular type of skin sensitization may persist indefinitely.

It is true that, in both untreated and poorly-treated patients, gummata may heal only to have new gummata develop subsequently. In such cases, however, there is no proof that the healing and subsequent development of a new gumma were caused by an alteration in the skin sensitivity. Healing may have been due to the death of treponemes in the lesion and new gummata may have developed when organisms again lodged within the skin. The organisms may have been brought to the skin by the blood stream or lymphatics or they may have penetrated from without. In any case, we cannot arbitrarily assume a rhythmic alteration of skin sensitivity in individuals who have relapses in the form of gummata.

The point I wish to make from the foregoing discussion is that syphilitic lesions of the skin and mucous membranes depend on at least two factors: the presence of $T$. pallida in the tissues and the reactivity of the tissues. In cases where early or late lesions heal only to have new lesions subsequently develop, both factors must be considered as well as very obscure immune mechanisms in the body.
This statement is at least true for the skin and mucous membranes. Whether or not it is equally true of all tissues is less certain. To my knowledge, no one has found $T$. pallida in the posterior columns of the spinal cord of tabetics nor in the corneae of patients with interstitial keratitis. Such phenomena as these and as general paresis, with its abundance of demonstrable organisms, raise numerous problems. All syphilitic lesions probably fall under the general heading of allergy, when this term is defined as altered reactivity of tissues to a specific antigen, and they afford an exceptionally comprehensive illustration of how little we know about the mechanisms underlying the great variety of "allergic" manifestations.

This still leaves unexplained the mechanism of acquired resistance to infection. If humoral antibodies are not notably protective, is there such a thing as acquired tissue immunity in the sense that treponemes are destroyed by tissues such as the skin and mucous membranes? Why, for example, should some of the Sing Sing volunteers, previously treated for late syphilis, develop evidence of infection after inoculation while others did not? All had positive TPI titres when inoculated. Presumably the inoculated treponemes multiplied in the infected group after inoculation, but not in the group with no evidence of infection. In the long history of acquired resistance to a specific antigen, a variety of immune mechanisms have undoubtedly evolved. Humoral antibodies probably play some role in acquired immunity to syphilis, but other older or newer mechanisms must also participate.

Because of our failure to understand the immune mechanisms in syphilis, and because of the chronicity and allergic nature of the disease, I have long been sceptical about the practical possibilities of a vaccine. If the infection itself does not produce resistance to re-infection after cure of the early stage, which may last 2 years or longer, why should a vaccine succeed? Possibly a course of vaccines might desensitize the skin and mucous membranes so that early lesions would not develop, but that would not rule out the possibility of asymptomatic infection. It is even conceivable that a course of yearly vaccines might produce in some individuals the sensitization characteristic of gummatous reactions. This hypothesis may be far fetched, but a more serious possibility is that vaccines capable of producing immunity to infection might cause, at least for some time, the production of positive serological tests for syphilis. In that case, the diagnosis of syphilis would be complicated still further.

In spite of these objections, I hope that efforts to develop a vaccine will be made. My objections may 
be ill founded, and even if they are valid a vaccine might be a useful tool in experiments designed to elucidate some of the unsolved problems I have mentioned. The fact that the once formidable spirochaete is now pretty much at our mercy, when we find it, is no reason, in my opinion, to abandon efforts to uncover its secrets and those of its human host.

\section{REFERENCES}

Chesney, A. M. (1927). "Immunity in Syphilis". Williams and Wilkins, Baltimore. Reprinted from Medicine (Baltimore), 5, 463. (1926).

Gjestland, T. (1955). "The Oslo Study of Untreated Syphilis-An Epidemiologic Investigation of the Natural Course of Syphilitic
Infection as based on a Restudy of the Boek-Bruusgard

Material"". Acta derm.-venereol. (Stockh.), 35, suppl. 34. Report on a Mass-treatment Campaign in Bosnia". WHO Monograph Series No. 11, WHO, Geneva.

Langer, E. (1926). Quoted by Stokes, J. H., Beerman, H., and Ingraham, N. R. Jr. (1944). "Modern C̈linical Syphilology", 3rd ed., p. 896, Saunders, Philadelphia.

Magnuson, H. J., Thomas, E. W., Olansky, S., Kaplan, B. I., DeMello, L., and Cutler, J. C. (1956). Medicine (Baltimore), $35,33$.

- Thompson, F. A., Jr., and McLeod, C. P. (1951). J. Immunol., 67,41 .

Moore, J. E. (1941). "The Modern Treatment of Syphilis". 2nd ed., p. 25, Thomas, Springfield, IIl.

Rosahn, P. D. (1947). "Autopsy'Studies in Syphilis, A Monograph." J. Vener. Dis. Inform., suppl. 21. U.S. Public Health Service, Washington, D.C.

Stokes, J. H., Beerman, H., and Ingraham, N. R. Jr. (1944). "Modern Clinical Syphilology," 3rd ed., pp. 770-771. Saunders, Philadelphis.

Thomas, E. W. (1949). "Syphilis: Its Course and Management". Macmillan, New York. 